\title{
Formal Barriers to Longest-Chain Proof-of-Stake Protocols ${ }^{*}$
}

\author{
JONAH BROWN-COHEN, KTH Royal Institute of Technology \\ ARVIND NARAYANAN ${ }^{\dagger}$, Princeton University \\ ALEXANDROS PSOMAS, Carnegie Mellon University \\ S. MATTHEW WEINBERG ${ }^{\ddagger}$, Princeton University
}

The security of most existing cryptocurrencies is based on a concept called Proof-of-Work, in which users must
solve a computationally hard cryptopuzzle to authorize transactions ("one unit of computation, one vote").
This leads to enormous expenditure on hardware and electricity in order to collect the rewards associated
with transaction authorization. Proof-of-Stake is an alternative concept that instead selects users to authorize
transactions proportional to their wealth ("one coin, one vote"). Some aspects of the two paradigms are the
same. For instance, obtaining voting power in Proof-of-Stake has a monetary cost just as in Proof-of-Work:
a coin cannot be freely duplicated any more easily than a unit of computation. However some aspects are
fundamentally different. In particular, exactly because Proof-of-Stake is wasteless, there is no inherent resource
cost to deviating (commonly referred to as the "Nothing-at-Stake" problem).
In contrast to prior work, we focus on incentive-driven deviations (any participant will deviate if doing so
yields higher revenue) instead of adversarial corruption (an adversary may take over a significant fraction of
the network, but the remaining players follow the protocol). The main results of this paper are several formal
barriers to designing incentive-compatible proof-of-stake cryptocurrencies (that don't apply to proof-of-work).

CCS Concepts: • Theory of computation $\rightarrow$ Cryptographic protocols; Algorithmic game theory; • Applied computing $\rightarrow$ Digital cash.

Additional Key Words and Phrases: cryptocurrencies; proof of stake; longest chain

ACM Reference Format:

Jonah Brown-Cohen, Arvind Narayanan, Alexandros Psomas, and S. Matthew Weinberg. 2019. Formal Barriers to Longest-Chain Proof-of-Stake Protocols. In ACM EC '19: ACM Conference on Economics and Computation (EC '19), fune 24-28, 2019, Phoenix, AZ, USA. ACM, New York, NY, USA, 15 pages. https://doi.org/10.1145/ 3328526.3329567

\section{INTRODUCTION}

Since Nakamoto's white paper in 2008 [19], Bitcoin and other cryptocurrencies have become ubiquitous, with hundreds of billions of USD worth of various cryptocurrencies currently in circulation. ${ }^{1}$ While existing technology is already remarkable, it remains an active research area on many fronts. This paper focuses on one aspect of this agenda: Proof-of-Work versus Proof-of Stake. We provide a brief overview of the salient aspects of cryptocurrencies below before highlighting our contributions.

\footnotetext{
${ }^{*}$ A full version of this paper can be found in [3]

$\dagger$ Supported by NSF CNS-1651938 and a grant from the Ripple University Blockchain Research Initiative.

¥Supported by NSF CCF-1717899 and a grant from the Ripple University Blockchain Research Initiative.

${ }^{1}$ https://coinmarketcap.com
}

Permission to make digital or hard copies of all or part of this work for personal or classroom use is granted without fee provided that copies are not made or distributed for profit or commercial advantage and that copies bear this notice and the full citation on the first page. Copyrights for components of this work owned by others than the author(s) must be honored. Abstracting with credit is permitted. To copy otherwise, or republish, to post on servers or to redistribute to lists, requires prior specific permission and/or a fee. Request permissions from permissions@acm.org.

EC '19, June 24-28, 2019, Phoenix, AZ, USA

(C) 2019 Copyright held by the owner/author(s). Publication rights licensed to ACM.

ACM ISBN 978-1-4503-6792-9/19/06...\$15.00

https://doi.org/10.1145/3328526.3329567 


\subsection{What is a Cryptocurrency?}

At their core, all cryptocurrencies are simply decentralized ledgers. A network of participants wish to agree upon a sequence of events and the order in which they occurred. These events could be monetary transactions in the case of Bitcoin, script commands in the case of Ethereum, or many others. Two salient features of cryptocurrencies that define their purpose are the following:

- It is crucial that the entire network reach consensus on the occurrence of events. For instance, a currency isn't very useful if users can't agree on who owns which coins. A shared virtual machine isn't very useful if users can't agree on its state.

- The network is permissionless and pseudonymous. That is, no identification outside the network is necessary to join and participate. ${ }^{2}$

Basic cryptography ensures that users can't forge transactions from other accounts, or undetectably propose otherwise invalid commands, so the main challenge is ensuring that users all agree on a state of the ledger, and also that no adversary can unduly influence the ledger's state, or otherwise gain by subverting the protocol. The typical attack to keep in mind is called a "double-spend:" imagine that you wish to purchase a car with Bitcoin. You digitally sign a transaction paying the owner a large sum of Bitcoin and broadcast it. The network agrees on a state of history where this transaction occurs, and then you get the car keys. As soon as this happens, you announce a digitally signed transaction that pays the same Bitcoin to an alternate account that you control, and do your best to subvert the protocol to agree on a ledger that includes this new transaction instead, leaving you with your Bitcoin intact as well as your new car. ${ }^{3}$ The point here is that the ledger is the currency: if the network believes that a transaction didn't take place, then by definition that transaction didn't take place.

A strawman proposal to cope might sound like this: every ten minutes, a uniform random participant from the network is selected. They may output a list of any number of consistent, valid transactions they like, and broadcast this to the entire network, along with a hash pointer to a previous selectee's output. Each output is assigned some numerical score, ${ }^{4}$ and users are asked to "believe" the history associated with the highest-scoring output they've seen so far. The key problem with this proposal is that selecting a uniformly random participant in a permissionless environment is absurd: users can freely create many "Sybils", making a uniformly random participant simply the participant who successfully created the most IDs. One of the key ingredients in successful cryptocurrencies is a random selection process that is Sybil-proof.

Proof-of-Work Versus Proof-of-Stake. The two most popular approaches to the above challenge are termed "Proof-of-Work" and "Proof-of-Stake." Proof-of-Work is employed by Bitcoin and Ethereum, and aims to select a user randomly, but proportional to their computational power. The idea is that while an attacker can certainly go out and purchase more computational power, exaggerating your computational power comes at a cost (unlike creating additional IDs). Proof-of-Work is typically implemented by requiring that all messages are concatenated with a nonce such that HASH(message, nonce) $<<2^{256}$, for some ideal hash function HASH with 256-bit output. It is widely believed that the best way to find such a nonce is to randomly guess (referred to as mining), and so every unit of computational power gives you a small additional probability of being able to send a valid message. As a result, some estimates predict over 2 billion USD spent annually

\footnotetext{
2"Permissioned blockchains" are becoming popular in finance, but these are fundamentally different than permissionless cryptocurrencies.

${ }^{3}$ Note that no ledger can possibly contain both transactions, as they spend the same coins. A currency would have no use if it were possible to spend the same coins twice.

${ }^{4}$ For instance, Bitcoin's "longest-chain rule" (essentially) assigns a score to output B equal to B's distance from the root (when following hash pointers). See Definition 4 in Section 2.
} 
(between electricity, cooling, etc.) just computing hashes (more than a fourth of the NSF budget for 2017) [20].

Proof-of-Stake isn't as widespread, but is an ongoing research focus of Ethereum [4], and is still responsible for billions of USD through cryptocurrencies such as NEM [8], Cardano, ${ }^{5}$ BlackCoin [25], PeerCoin [18], Nxt [7], and Tezos [14]. The goal is to select a user randomly, and proportional to their wealth (in the currency itself). The idea again is that while an attacker can certainly go out and purchase more stake in the currency, it comes at a cost. In comparison to Proof-of-Work, Proof-of-Stake wastes no electricity.

\subsection{Security Concerns Specific to Proof-of-Stake}

From a security perspective, it's actually convenient that Proof-of-Work wastes resources: this guarantees that certain deviations (discussed in the next paragraph) from the intended protocol also cost additional resources, and are naturally disincentivized (this, of course, does not mean that deviations are never profitable, see e.g. [5, 10, 11, 23]). Proof-of-Stake, on the other hand, has the property that such deviations consume no resources and this is exactly because Proof-of-Stake is wasteless, so it falls on the protocol to disincentivize such behavior through clever reward schemes.

This phenomenon is commonly referred to as "Nothing-at-Stake," and refers to the fact that it consumes no additional resources for participants to, for instance, copy an outdated history of the currency and participate simultaneously with the "real" one, or even to copy every outdated history and participate in all of them simultaneously. Numerous recent works, both commercial and academic, aim to address this challenge with clever reward schemes. Commercial protocols indeed propose interesting approaches, and don't seem to have suffered major security setbacks to date. However, these ideas appear informally in whitepapers, often without formal definitions or rigorous reasoning, and are far from being fully explored. Academic proposals, on the other hand, provide rigorous, provable security guarantees, usually in "network intrusion" models (where a fraction of the network is malicious, but the remainder is honest).

In contrast to both existing streams of literature, we focus on rigorous guarantees in a "strategic" model ( $100 \%$ of users act to maximize their own reward). The main result of this paper is a formal barrier to incentive-compatible proof-of-stake protocols. Specifically, we introduce a model for Proof-of-Stake protocols that captures the vast majority of commercial and academic proposals. Next, we show that every protocol fitting into this model must satisfy one of two complementary properties. Finally, we show how an attacker can exploit each one of these properties in order to benefit by strategically deviating from the prescribed protocol.

At a conceptual level, the barriers stem from the following: all cryptocurrencies require some source of (pseudo)randomness. In Proof-of-Work, this pseudorandomness is in some sense external to the cryptocurrency: the first miner to successfully find a good nonce produces the next block, and this miner is selected completely independently of the current state of the cryptocurrency.

In Proof-of-Stake, it is highly desirable that the pseudorandomness comes from within the cryptocurrency itself, versus an external source (due to network security concerns discussed in Section 2). One might initially suspect that with sufficiently many hashes or digital signatures of past blocks, this can indeed serve as a good source of pseudorandomness for future blocks. However, we formalize surprising barriers showing a fundamental difference between external pseudorandomness and pseudorandomness coming from the cryptocurrency itself.

\footnotetext{
$\overline{{ }^{5} \text { https://www.cardano.org/en/home/ }}$
} 


\subsection{Our Model: Formal Guarantees in Ideal Network Conditions}

Seminal work of Fischer, Lynch, and Paterson [12] proves that if one desires formal consensus guarantees, even in the presence of a single adversarial user, one must make some network assumptions. Existing academic works therefore aim to make the minimal assumptions necessary.

Consider instead an ideal network where every node has perfectly synchronized clocks, and every message is received with zero latency by every other node. It is not hard to design secure Proof-of-Stake cryptocurrencies in this model (at least, ones that are secure against known attacks), but these protocols will look completely unrealistic. The key power that this assumption buys is the ability to ignore messages that arrive even slightly late, as they cannot have been sent by an honest participant. The problem with targeting a solution that is only secure in this ideal model is that you wind up with an extremely fragile protocol which can't handle even minimal latency.

Still, despite how unrealistically strong this model is, the authors aren't aware of existing protocols without incentive issues even in this ideal network model (put another way, it is surprisingly daunting to develop a protocol that is secure in the typical sense for any non-trivial network model, yet secure in the strategic sense in the ideal network model). Rather than posing a long list of similar looking attacks, we formalize intuitively undesirable properties of a Proof-of-Stake protocol that capture the issues in many existing proposals. For each property, we show that any Proof-of-Stake protocol with this property is vulnerable to a certain kind of attack. We elaborate further on these properties and attacks in the technical sections.

\subsection{Comparison to Related Work}

The "incentive-driven" threat to cryptocurrencies appears well-understood even as far back as Nakamoto's whitepaper, yet has received considerably less formal attention. Some notable exceptions unrelated to Proof-of-Stake include [2, 5, 10, 11, 16, 23]; also see [15] for a survey ca 2018. While such "attacks" aren't a direct threat to consensus, they pose a severe indirect threat: [11] observes that such attackers gobbling up profits from honest participants could drive them out of the market, enabling a threat to consensus.

The most obviously related works to the present paper are academic Proof-of-Stake proposals [9, $13,17]$. These works focus primarily on the network intrusion threat model, as it is obviously important that proposals be secure in the classical sense before concerning oneself with incentives.

Some works go further and provide incentive guarantees. Saleh [22] shows that following the longest chain protocol is an equilibrium in a model where miners have discounted utilities and receive rewards only once consensus is reached. Daian et al. [9], Kiayias et al. [17] prove that miners who strategically deviate from the prescribed protocol can only gain a small $\varepsilon$ fraction of the total rewards. Still, [9] notes that it is preferable for known strategic deviations to be strictly disincentivized (and prove that their scheme achieves this for Nothing-at-Stake), and [17] observes that not all known deviations are captured by such claims (and prove that their scheme successfully disincentivizes double-spending). In the context of these works, we propose that the deviations formalized in this paper receive similar treatment to currently-known attacks in future analyses. We provide much more detail regarding how our results interact with existing proposals in the full version of this paper. We also wish to emphasize here that protocols which fit our model (and therefore are vulnerable to the attacks we develop) are currently deployed, and new ones are still being developed. We are of course not claiming that the attacks we discover are devastating (after all, Bitcoin has always been vulnerable to selfish mining, but never suffered an attack), but that they should certainly be addressed by future designs (and we discuss approaches to do so in Section 5). 


\subsection{Summary of Contributions and Roadmap}

Our model aims to isolate the incentive-driven threat, and we formalize two complementary properties such that every longest-chain Proof-of-Stake protocol must satisfy one of them. We further demonstrate incentive-driven attacks against protocols satisfying either of these properties.

Section 2 contains necessary definitions related to cryptocurrencies and Proof-of-Stake (where even our formal definition of Proof-of-Stake may be of interest). Section 3 defines generic properties shared by a wide class of protocols. Section 4 poses generic attacks against any protocol with the properties defined in Section 3. Section 5 provides more detailed context for our paper with respect to prior work and proposes future directions.

\section{PRELIMINARIES}

Definition 1 (Block). A cryptocurrency stores its decentralized ledger in a set of objects called blocks. Every block $B$ contains a pointer to its predecessor $P_{R E D}(B)$, a previous block (via its HASH). Every block is created by a single miner, denoted by $\operatorname{MINER}(B)$, and has a timestamp $t_{B}$ that indicates its claimed creation time (that is, the creator of the block can insert any value they like for $t_{B}$, independent of the actual time at which it was created). Blocks also contain some other information that has semantic meaning (such as transactions in the case of Bitcoin, scripts in the case of Ethereum, etc.).

Each block $B$ describes a potential history of events, as defined by the semantic contents of $B$ and its predecessors. For example, a Bitcoin block describes a series of monetary transactions.

Definition 2 (Coin). The basic monetary unit of any cryptocurrency is called a coin, referenced by a unique ID. Every coin has an owner, referenced by a unique public key. Certain transactions have an associated semantic meaning that changes the owner of a coin. So for a given block B, and coin c, one can define $\mathrm{OWNER}_{B}(\mathrm{c})$ to be the owner of a coin $c$ as defined by the semantic meaning of transactions included in the history defined by block B. Here, we are referring to the smallest discrete monetary unit (so one Satoshi in the case of Bitcoin, rather than one bitcoin).

The key problem that all cryptocurrencies need to resolve is the consensus problem: how can we get all nodes in the network to eventually agree on the occurrence of events? All such protocols necessarily have a notion of validity. That is, users cannot just generate arbitrary messages and send them at arbitrary times and have the rest of the network recognize these as potential blocks to be added to history (for instance, the block could contain invalid transactions, or it might not be that user's "turn" to send a message). We focus on protocols that satisfy the following assumptions:

Assumption 1. Let $T(B)$ be the graph whose nodes are blocks who share an ancestor with $B$, and whose edges are pointers to predecessors. We assume that:

(a) (Chain Dependence) Block B's validity at time $t$ only depends on B, $t$ and B's predecessors.

(b) (Monotonicity) If a block $B$ is valid at time $t$ for a given graph $T(B)$, then it is valid for all graphs $T^{\prime}$ that contain $T(B)$ as a subgraph and for all times $t^{\prime} \geq t$.

Before continuing, let's quickly motivate/discuss Assumption 1. All protocols that the authors are aware of satisfy Assumption 1, and this is for good reason: all protocols are vulnerable to what are called "Eclipse" attacks. An Eclipse attack occurs when an adversary prevents or blocks messages to honest participants of the protocol. If an adversary can temporarily partition the network into disjoint sets, of course the network can't reach consensus while partitioned. However, one might hope that the network can reach consensus once reunited. A major barrier to this possibility would be if a user once believed block $B$ to be valid (and built a deep history on top of it), and only learned once reunited that in fact $B$ was invalid all along. Without Chain Dependence and Monotonicity, this situation is entirely possible. With Chain Dependence and Monotonicity, Eclipse attacks are 
still a threat, but at least they cannot trick a user into believing a block is valid only to discover later that it was in fact invalid.

To get some intuition for Assumption 1, here are two toy examples that violate Chain Dependence and Monotonicity (respectively). First, consider a protocol where the validity of a block depends on a trusted source of external randomness (e.g. the NIST beacon). This external randomness is not contained in the blockchain itself, so such a protocol does not satisfy Chain Dependence. Next, consider a protocol that declares a block $B$ invalid if its creator proposed another block $B^{\prime}$ within the same 2-week time period. Then $B$ will be considered valid for the graph that contains only $B$ and its predecessors, but invalid for the graph which contains $B, B^{\prime}$ and all of their predecessors. So this protocol violates Monotonicity. These protocols are not absurd, but are much more vulnerable to Eclipse attacks than virtually all existing proposals.

Now, we begin restricting attention to Proof-of-Stake protocols, in which the owner of a coin $c$ is eligible to mine a new block $B$ at some time $t$ according to the rules of the protocol. That is, every block $B$ further references a coin, $c_{B}$ that is used to witness $B$ 's validity. For any protocol satisfying Assumption 1, the validity of a block can be determined by a function that takes only a block $B$ and the current time $t$ as input. This is because all of $B$ 's predecessors can be accessed by following back the predecessor pointers to the root (and $t_{B}, c_{B}$ are both included in $B$ ). Thus, under these assumptions, a Proof-of-Stake Protocol can be defined as follows:

Definition 3 (Proof-of-Stake Protocol). A Proof-of-Stake Protocol $P$ is fully defined via two deterministic functions: a validating function $V_{P}$ and a mining function $M_{P}$. The validating function satisfies the following requirements:

- $V_{P}$ takes as input a block B (which includes the claimed time of creation, $t_{B}$, and the claimed coin witness, $\left.c_{B}\right)$, and outputs an element of $\{0,1\}$.

- $V_{P}$ must be efficiently computable by every participant in the protocol.

- A block $B$ is valid at time $t$ if and only if $\operatorname{PRED}(B)$ is valid and

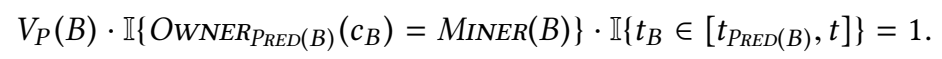

The mining function $M_{P}$ satisfies the following requirements:

- $M_{P}$ takes as input a block $A$, a coin c and timestamp $t$ and outputs a block.

- $M_{P}(A, c, t)$ is efficiently computable by $\mathrm{OWNER}_{A}(c)$.

- For any coin $c$ and any time $t$, if there exists a block $B$ such that $B$ is valid at time $t$, where $P_{R E D}(B)=A, c_{B}=c$, and $t_{B}=t$, then $M_{P}(A, c, t)=B^{\prime}$ where $B^{\prime}$ is valid, $t_{B^{\prime}}=t, c_{B^{\prime}}=c$, and $\operatorname{PRED}\left(B^{\prime}\right)=A$.

- For any coin $c$ and any time $t$, if there is no block $B$ such that $B$ is valid at time $t$, where $\operatorname{PRED}(B)=A, c_{B}=c$, and $t_{B}=t$, then $M_{P}(A, c, t)=\perp$.

Again before continuing, let's parse some aspects of this definition and what separates Proof-ofStake from Proof-of-Work. The first two $V_{P}$ bullets are uncontroversial: a block is valid or it isn't, and every user in the network better be able to tell which blocks are valid. The third bullet might at first appear confusing, but recall that $B$ contains a reference to $t_{B}$, the claimed time of creation, and $c_{B}$, the coin witnessing validity. So $V_{P}(\cdot)$ can in fact depend on these. Beyond that, the first indicator is necessary to ensure that miners can't cheat by moving the same coin around different public keys in a potential block in order to make that block itself valid. The second indicator is necessary to guarantee that a miner can't claim to have produced a block before s/he heard about its predecessor, nor claim to have produced a block in the future (but otherwise the current timestamp is irrelevant for determining a block's validity, due to Assumption 1).

As for $M_{P}$, the second bullet captures two salient features. The first is that $M_{P}(A, c, t)$ is efficiently computable. This is what separates Proof-of-Stake from Proof-of-Work: if $M_{P}$ were not efficiently 
computable (e.g. because it involved inverting an ideal hash function), it would require non-trivial work to mine (Proof-of-Work). Because $M_{P}$ is efficiently computable, the owner of coin $c$ need only run $M_{P}(A, c, t)$ once during timestep $t$ for each coin they own, and has nothing to gain by doing additional work. The second salient feature is actually an omission: that $M_{P}(A, c, t)$ is not necessarily efficiently computable by miners other than $\operatorname{OWNER}_{A}(c)$ (e.g. because it perhaps requires producing a digital signature). The last two bullets simple say that $M_{P}$ outputs some valid block, if one exists, and otherwise outputs $\perp$.

Remark 1. In the definition for Proof-of-Stake we provided, it may sometimes be desirable to "freeze" coins used to mine for longer than just one block. That is, the first indicator in bullet three requires that coin $c$ be owned by MINER(B) in the block before B (so that the miner could not move $c$ in the same block $s /$ he is trying to mine). For many protocols, it may be desirable to additionally insist that the owner of coin $c$ did not change in any of the last $F$ blocks (i.e. that $c$ was frozen for $F$ blocks before used for mining). We note that our definition can easily be modified to replace $\mathbb{I}\left\{\operatorname{OWNER}_{P_{R E D}(B)}\left(c_{B}\right)=\operatorname{MINER}(B)\right\}$ with $\mathbb{I}\left\{O W N E R_{P_{R E D}(B)}\left(c_{B}\right)=\operatorname{MINER}(B) \forall i \leq F\right\}$, where $F$ is a freezing parameter of $P$ (and Definition 3 is then a special case with $F=1$ hardcoded). Of course, this could also be offloaded into $V_{P}(\cdot)$, but the astute reader will later notice that treating freezing separately makes future technical definitions cleaner.

So far we have only discussed the validity of blocks. We also need to discuss where an honest user should mine (i.e. which block $A$ should be input to $M_{P}$ ). The dominating paradigm among existing proposals (including all commercial protcols referenced in Section 1, but excluding Algorand [6, 13], Casper [4], and GHOST [24]) are variants of the longest-chain protocol, where each block is given a monotone increasing score, and nodes are asked to "believe" the highest scoring block. ${ }^{6}$

Definition 4 (Longest-Chain Variant). A Longest-Chain Variant has an associated scoring function $S(\cdot)$. $S$ takes as input a block $B$, outputs a score $S(B) \in \mathbb{R}$, and is monotone increasing: if $B^{\prime}=P R E D(B)$, then $S(B)>S\left(B^{\prime}\right)$. A Longest-Chain-Variant consensus protocol associated with $S$ asks users to mine on the valid block $A$ maximizing $S(A)$ (among blocks they are aware of). ${ }^{7}$

Longest-Chain Variants are particularly common within cryptocurrencies because of their robustness to Eclipse attacks. Even if the network is partitioned for an extended period, and both disjoint subsets produce completely different histories, the entire network will quickly converge to the higher-scoring history as soon as the subsets reunite. Alternative protocols based on Byzantine Consensus [4, 6] lack this property, and instead achieve finality. That is, once a user considers a block $B$ to be included in the ledger, they will never consider valid any ledger that not including $B$. Indeed, in protocols with finality, if the network is partitioned for an extended period, progress will either stall, or the network will never reach consensus even after being reunited.

\section{PROPERTIES OF PROTOCOLS}

In this section we introduce two simple, intuitively desirable properties for Proof-of-Stake protocols.

\section{1 (Un)-Predictability}

The first desirable property we define is unpredictability. Intuitively, it is good for protocols to be unpredictable in the sense that miners do not learn that they are eligible to mine a block

\footnotetext{
${ }^{6}$ GHOST [24] technically cannot be phrased in this language because the score of a block depends on the existence of descendants of $B$ 's ancestors not referenced directly in $B$. If instead blocks are required to include pointers to these other blocks in order to "get credit," then GHOST would also fit in this language. See the full version of this paper for a further discussion of different concerns regarding GHOST and Proof-of-Stake.

${ }^{7}$ Ties are allowed to be broken arbitrarily, but consistently (i.e. if $S(A)=S\left(A^{\prime}\right)$, users can arbitrarily decide to adopt $A$ or $A^{\prime}$, but cannot switch between adopting $A$ and $A^{\prime}$ ).
} 
until shortly before it is due to be mined. Many attacks, such as double-spending (discussed in Section 1), or selfish-mining ([11], discussed in Section 4), can become much more profitable if miners know in advance when they become eligible to mine. We begin with the definition of local predictability, which describes protocols where the owner of a coin knows in advance if she is eligible to mine a block with that coin. Let $\operatorname{PRED}^{D}(B)$ be the $D$-th predecessor of a block $B$. That is, $\operatorname{Pred}^{1}(B)=\operatorname{Pred}(B)$ is the block that $B$ is mined on top of, $\operatorname{PreD}^{2}(B)=\operatorname{Pred}(\operatorname{Pred}(B))$, etc.

Definition 5 ( $D$-locally predictable). A coin $c$ is D-locally predictable at block $A$ for timestamp $t$ if $O W N E R(c)$ can efficiently predict whether or not there will exist a block $B$ with $c_{B}=c$ such that $V_{P}(B)=1$, where $\operatorname{PRED}^{D}(B)=A$ and $t_{B}=t$.

Observation 1. For any Proof-of-Stake protocol, every coin c is 1-locally predictable at every block $A$ for every timestamp $t>t_{A}$.

Proof. Fix a coin $c$, block $A$, and timestamp $t>t_{A}$. If there is some block $B$ with $c_{B}=c$ such that $V_{P}(B)=1, t_{B}=t$ and $\operatorname{PRED}^{D}(B)=A$, then $M_{P}(A, c, t)$ outputs such a block. If not, then $M_{P}(A, c, t)=\perp$. Since $M_{P}$ is efficiently computable by Owner $(c)$, we have that the coin $c$ is 1-locally predictable at block $A$ for timestamp $t$.

In many existing protocols, every coin in a given protocol will be $D$-locally predictable at every block and for every timestamp. In such cases, we will refer to the protocol itself as being D-locally predictable. Intuitively, local predictability captures that a miner can predict in advance when they will be able to produce a block (whereas in Proof-of-Work protocols, they learn only the instant that the block is produced). Global predictability is a stronger definition which describes protocols in which every participant knows in advance if the owner of a given coin is eligible to mine a block

Definition 6 ( $D$-globally predictable). A coin c is $D$-globally predictable at block A for timestamp $t$ if every participant of the protocol can efficiently predict whether or not there will exist a block $B$ with $c_{B}=c$ such that $V_{P}(B)=1$, where $\operatorname{PRED}^{D}(B)=A$ and $t_{B}=t$.

Below we provide examples of predictable and unpredictable protocols for the sake of further explanation. In what follows, let $A=\operatorname{PRED}^{D}(B)$ and $T \in \mathbb{R}$ be some positive threshold.

- Protocol $P_{1}$ where $V_{P_{1}}(B)=1$ if and only if $\operatorname{HASH}\left(A, t_{B}, c_{B}\right)<T{ }^{8}$ Every coin $c$ in $P_{1}$ is $D$-globally predictable at $A$, for all $D$ and all $A$, since every user can compute $\operatorname{HASH}\left(\operatorname{PrED}^{D-1}(A), t^{\prime}, c^{\prime}\right)$ for all $t^{\prime}, c^{\prime}$ and check whether or not it's $<T$. So every user can compute the minimum $t_{1}^{\prime}$ such that a block can be built on top of $A$. Similarly, every user can compute $\operatorname{HASH}\left(\operatorname{PRED}^{D-i}(A), t^{\prime}, c^{\prime}\right)$ for all $i \leq D$, which determines the minimum $t_{i}^{\prime}$ that a block can be built with $\operatorname{PRED}^{i}(B)=A$ (note that $t_{i}^{\prime}$ must be monotonically non-decreasing in $i$ ). If $t_{D}^{\prime}<t$, then a block $B$ indeed exists with $\operatorname{PRED}^{D}(B)=A, c_{B}=c, t_{B}=t, V_{P_{1}}(B)=1$. Otherwise, no such $B$ exists.

- Protocol $P_{2}$ where $V_{P_{2}}(B)=1$ if and only if $\operatorname{HASH}\left(t_{B}, c_{B}\right)<T$. Every coin $c$ in $P_{2}$ is $D$-globally predictable at $A$ for all $D$ and all $A$. This is because every user can compute $\operatorname{HASH}\left(t^{\prime}, c^{\prime}\right)$ for all coins $c^{\prime}$ and times $t^{\prime}$. Therefore, every user can determine all potential timesteps where a block could be created. If there are $\geq D$ such timesteps between $t_{A}$ and $t$, and $\operatorname{HASH}(t, c)<T$, then the answer is yes. If not, then the answer is no.

- Protocol $P_{3}$ where: ${ }^{9}$

- Each block $B$ contains a signature $s_{B}$ computed by $\operatorname{MineR}(B)$.

- $M_{P_{3}}(B, c, t)$ outputs a block $B$ with $s_{B}=\operatorname{SIG}_{\mathrm{OWNER}(c)}\left(\operatorname{HASH}\left(s_{A}\right), t\right)$, where $\operatorname{SIG}_{\mathrm{OwNER}(c)}(\cdot)$ denotes the function which digitally signs a message using the secret key of OwNER $(c)$.

\footnotetext{
${ }^{8}$ Note that this is (essentially) the initial proposal made in Peercoin: https://peercoin.net/.

${ }^{9} P_{3}$ is based on the seed-selection portion of Algorand.
} 
$-V_{P_{3}}(B)=1$ if and only if $\operatorname{HASH}\left(s_{B}\right)<T$.

$P_{3}$ is a good example to clarify potentially subtle aspects of the definitions. Every coin in $P_{3}$ is 1-locally predictable at $A$, for all $A$ (as with all Proof-of-Stake protocols). In addition, Owner $(c)$ can computationally efficiently find certain kinds of blocks $B$ with $t_{B}=t$ and $\operatorname{PRED}^{D}(B)=A$ : namely, those for which $\operatorname{Miner}\left(B^{\prime}\right)=\operatorname{Owner}(c)$ for all $B^{\prime}=\operatorname{PRED}^{i}(B)$ for $i \leq D$. This is because OWNer $(c)$ can efficiently compute all blocks $B^{\prime}$ that they themselves can build on top of $A$, and then all blocks that they themselves can build on top of these blocks, etc. using $M_{P_{3}}$. However, OwNER $(c)$ cannot computationally efficiently predict whether there exists a block $B$ with $\operatorname{Pred}^{D}(B)=A, t_{B}=t$, but $\operatorname{MineR}\left(\operatorname{Pred}^{i}(B)\right) \neq \operatorname{Owner}(c)$ for some $i<D$. This is because knowing the existence of this block would require being able to digitally sign as $\operatorname{MinER}\left(\operatorname{PrED}^{i}(B)\right)$, which Owner $(c)$ cannot do computationally efficiently. So every coin $c$ in $P_{3}$ is not $D$-locally predictable for any $D>1$. Similarly, no coin is $D$-globally predictable for any $D$ because in order to know whether $B$ is valid, one must be able to digitally sign messages as $\operatorname{OwnER}\left(c_{B}\right)$ (which one cannot do computationally efficiently unless one is $\left.\operatorname{OwnER}\left(c_{B}\right)\right)$.

\section{2 (Non)-Recency}

The second property we consider is recency, which is just the negation of local predictability. Intuitively, a protocol is $D$-recent at $A$ if the validity of block $A$ depends on some information contained in the last $D$ predecessors of $A$. The main security concern with $D$-recent protocols is that intuitively each chain has its own pseudorandomness (but this is not a formal claim) ${ }^{10}$ We'll again get into more detail with respect to security implications in Section 4, but just note here that certain deviations are easier to detect when chains share the same pseudorandomness.

Definition 7 (D-recent). A coin $c$ is D-recent at a block $A$ for timestamp $t$ if the owner of $c$ cannot efficiently predict whether or not there will exist a block $B$ such that $V_{P}(B)=1$, where $\operatorname{PRED}^{D}(B)=A$, $c_{B}=c$, and $t_{B}=t$.

As with predictability, in many existing protocols every coin will be $D$-recent at every block and for every timestamp. We will refer to such examples as $D$-recent protocols. Due to the following observation, further examples illustrating recency aren't necessary, as it's simply the negation of $D$-local predictability.

Observation 2. For any $D$, any block $A$ and any timestamp $t$, a coin $c$ is either D-locally predictable or D-recent.

\section{SECURITY IMPLICATIONS}

In this section, we elaborate on the security implications of predictability and recency.

\subsection{Global Predictability}

Here, we'll describe two attacks against protocols with coins that are globally predictable that we call "globally predictable selfish-mine" and "globally predictable double-spend." In the former, the attacker attempts to claim extra mining rewards by delaying the announcement of mined blocks, and in the latter the attacker attempts to receive goods for free by overwriting a transaction in order to effectively spend the same coins twice. Both attacks are also possible, but weaker and a touch more complex, against locally predictable protocols. So we begin with the globally predictable versions, and defer the locally-predictable variants to the full version of this paper. Both attacks have a similar flavor, so we detail selfish mining here, and also defer double spending to the full version.

\footnotetext{
${ }^{10}$ By chain, we mean a set of blocks $\left\{\operatorname{PrED}^{i}(B) \mid i \geq 0\right\}$.
} 
Definition 8 (Globally-Predictable Selfish Mining).

(1) At all times $t$, let $A$ denote the current longest chain (that is, let $A$ be the block you are aware of maximizing $S()$.$) .$

(2) For all $k>0$, find the minimum time $t_{k}^{\prime}$ such that there exists a block $B$, where $\operatorname{PRED}^{D}(B)=A($ for some $D>0), V_{P}(B)=1$, you own coin $c_{P_{R E D}(B)}$ for all $i \in[0, D-1], t_{B}=t_{k}^{\prime}$, and $S(B)>S(A)+k$. That is, for all $k$, find the earliest time that you can create a block $B$ with $S(B)>S(A)+k$, where you created all blocks on the path from $A$ to $B$.

(3) For all $k>0$, find the minimum time $t_{k}^{*}$ such that there exists a block $B$, where $\operatorname{PRED}^{E}(B)=A$ (for some $E>0), V_{P}(B)=1$, you don't own coin $c_{P_{R E D}{ }^{i}(B)}$ for all $i \in[0, E-1], t_{B}=t_{k}^{*}$, and $S(B)>S(A)+k$. That is, for all $k$, find the earliest time that the rest of the network can create a block $B$ with $S(B)>S(A)+k$, and you did not create any blocks on the path from $A$ to $B$.

(4) If at time $t$, there exists a $k$ such that $t_{k}^{\prime}<t_{k}^{*}$, immediately stop publishing blocks until $t_{k}^{\prime}$ (if there are multiple such $k$, take the largest one). At time $t_{k}^{\prime}$, output the promised B and its predecessors.

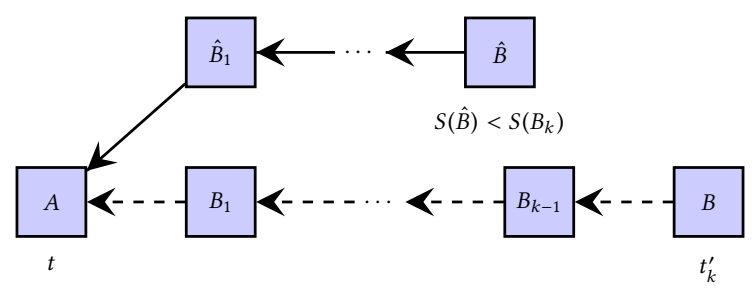

Fig. 1. Globally Predictable Selfish Mining (illustrated with $S(B)=$ number of predecessors of $B$ ): If you know you can make a node $B$ at time $t_{k}^{\prime}$ whose predecessors after $A$ are also all created by you, and $S(B)>S(\hat{B})$ for all possible $\hat{B}$ that could be created by time $t_{k}^{\prime}$ without you (whose predecessors after $A$ are also all created without you), stop publishing blocks and output the full chain $B_{1} \rightarrow \ldots \rightarrow B$ all at once at time $t_{k}^{\prime}$.

The high-level idea behind the attack is the following: the original selfish mining attack [11] proposes withholding a block $B$ upon creation (i.e. not broadcasting it). You continue mining on top of $B$, while the rest of the network continues mining on top of $\operatorname{PRED}(B)$. If you create a new block $B^{\prime}$ on top of $B$ before the rest of the network creates a new block on top of $\operatorname{PRED}(B)$, then you now possess the unique longest chain. So you can continue mining on top of $B$ and its descendants until the rest of the network finds a chain that is almost as long as yours. At this point you can announce your chain, causing the entire chain built by the rest of the network to be orphaned (because they will all adopt your uniquely longest chain). Of course, the attack could go completely differently: maybe the rest of the network successfully mines on top of $\operatorname{PRED}(B)$ before you mine on top of $B$. In this case, now you're in trouble and run the risk of losing $B$ because there is a competing chain of the same length.

With sufficient global predictability, however, there is no risk! You can predict before deciding whether to withhold $B$ if you'll mine on top of $B$ before another miner mines on top of $\operatorname{PRED}(B)$. So you can only withhold those $B$ for which the attack will succeed, completely avoiding the risk. For instance, if a protocol is $D$-globally predictable, and $S(B)=\#$ predecessors of $B$, then the above attack can be carried out for any $k \leq D$. The attacker's incentives to carry out such an attack of course depend on exactly how minng rewards are distributed, but it is clear that globally predictable selfish mining allows the attacker to produce a greater fraction of blocks on the longest chain. For standard reward schemes this is indeed profitable $[5,11]$.

The frequency with which an attacker will have the ability to predictably selfish-mine depends on the exact nature of the scoring function $S$ and the Proof-of-Stake protocol $P$. In the full version 
of this paper, we analyze the probability of a miner being able to launch a predictable selfishmining or predictable double spend attack assuming that $S(\cdot)$ is the simple longest-chain rule and that $P$ acts as a random oracle (formal definition in the full version - without some assumption like this it's impossible to begin talking about probabilities). The key takeaway from this section is that Predictable Longest-Chain Variant Protocols are vulnerable to Predictable Selfish Mining.

\subsection{Recency}

Here, we discuss an attack on $D$-recent protocols, which we call Undetectable Nothing-at-Stake. In the Nothing-at-Stake attack miners try to mine on top of many blocks simultaneously, instead of just the one maximizing $S(B)$ over all valid blocks. We call it undetectable if, information theoretically, there is no proof that a miner engaged in Nothing-at-Stake. To make this formal, it is helpful to first recall the behavior of an honest miner.

Definition 9 (Honest Miner). An Honest Miner participating in a Proof-of-Stake protocol will do the following at every time step $t$ :

- Find A maximizing $S(A)$ among all blocks that the miner is aware of.

- For all owned coins $c$, attempt to mine a new block $B=M_{P}(A, c, t)$. If $B \neq \perp$ announce the new block B, otherwise do nothing.

Any deviation from this behavior would be considered dishonest, and problematic for the functionality of the underlying protocol. Sometimes, these deviations will be detectable, in the sense that there is clear evidence that a miner deviated from the protocol. Formally:

Definition 10 (Provable Deviation). We say that two valid blocks $B$ and $B^{\prime}$ with $c_{B}=c_{B^{\prime}}=c$ are a provable deviation by the owner of coin $c$ if $t_{B^{\prime}}=t_{B}$, or if both $t_{B^{\prime}}>t_{B}$ and $S(B)>S\left(P_{R E D}\left(B^{\prime}\right)\right)$.

The first part of this definition captures that honest nodes only output one block per timestep. The second half captures that at time $t_{B}$, the owner of coin $c$ created block $B$. Then at time $t_{B^{\prime}}>t_{B}$, they are claiming that $\operatorname{PRED}\left(B^{\prime}\right)$ maximizes $S(\cdot)$ over all blocks they are aware of. Clearly this is not true if $S(B)>S\left(\operatorname{PrED}\left(B^{\prime}\right)\right)$. The proof of Proposition 1 can be found in the full version of this paper.

Proposition 1. If a miner is caught having announced a provable deviation, then they must have deviated from the intended protocol (i.e. there are no "false positives"). Also, any sequence of announcements from a miner that does not contain a provable deviation could have been sent by an honest miner experiencing latency.

Proposition 1 tells us that without a provable deviation, we cannot "punish" suspected deviant miners without the risk of punishing honest but poorly-connected miners. Now, we propose one specific protocol deviation that is guaranteed never to produce a provable deviation.

Definition 11 (Undetectable Nothing-at-Stake). First, ensure that all of your coins are owned by different public keys. Then, for each owned coin $c$, do the following during every timestep $t$ :

- Find A maximizing $S(A)$ among all blocks that you are aware of.

- Find $A^{\prime}$ maximizing $S\left(A^{\prime}\right)$ among all blocks that are not descendants of $P_{R E D}{ }^{D}(A)$.

- Mine $B=M_{P}(A, c, t)$ with $\operatorname{PrED}(B)=A$, and $B^{\prime}=M_{P}\left(A^{\prime}, c, t\right)$ with $\operatorname{PrED}\left(B^{\prime}\right)=A^{\prime}$.

- If $B \neq \perp$, announce the new block $B$. If $B^{\prime} \neq \perp$, and announcing $B^{\prime}$ would not create a provable deviation, announce $B^{\prime}$.

By definition, announcing $B$ and $B^{\prime}$ at time $t$ does not create a provable deviation at time $t$. But, it could be the case that at some later time $t^{\prime}>t$, announcing some new block $C$ would reveal that the miner was not following the honest protocol. We show next that this is impossible. 


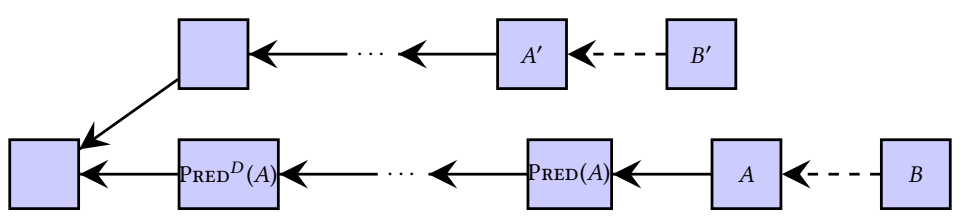

Fig. 2. Undetectable Nothing-at-Stake: The attacker creates two blocks, $B$ and $B^{\prime}$. There is no resource cost in checking if both are valid. Depending on reward scheme, there may be an expected monetary gain for announcing both.

Observation 3. The Undetectable Nothing-at-Stake strategy never produces a provable deviation.

Note that there is an explicit check before announcing any $B^{\prime}$ which doesn't build on top of the longest chain. In order to guarantee that such a $B^{\prime}$ doesn't cause a provable deviation, the miner checks that $S\left(\operatorname{PrED}\left(B^{\prime}\right)\right)>S(C)$, where $C$ is the most recent block she has mined. Therefore, the proposed deviation will never "get caught" (that doesn't necessarily mean that a clever protocol can't still enact punishment - see Section 5). But we also want to understand whether the proposed deviation will ever actually happen; it's conceivable that the "safety check" will prevent the miner from ever announcing an "illegitimate" block. This is where recency comes in. If a protocol is $D$ Recent, then whether or not a you can build a valid block on top of $A^{\prime}$ with coin $c$ at time $t$ depends on some of the blocks between $A^{\prime}$ and $\operatorname{PRED}^{D}(A)$. But, by Chain Dependence + Monotonicity, the validity of any block built on top of $A$ does not depend on any of the blocks between $A^{\prime}$ and $\operatorname{PRED}^{D}(A)$. Thus each coin $c$ is, in some sense, getting a "fresh shot" at being eligible to mine a block on top of $A^{\prime}$ during time $t$, and one might reasonably expect this shot to succeed with non-zero probability. In general, this success probability will depend on the exact behavior of $V_{P}(\cdot)$.

Of course, any meaningful probabilistic analysis requires some assumption on $S$ and $P$ (otherwise we don't even have a probability space to work with). In this paper we consider the case where $S$ is the simple longest chain rule and $P$ acts as a random oracle, and show essentially that when the number of coins in the system is larger than the recency of the protocol, then Undetectable Nothing-at-Stake is announcing twice as many blocks as the honest strategy. See the full version of this paper for a formal statement.

Again, the profitability of Undetectable Nothing-at-Stake depends on the exact reward scheme, but what is clear is that Undetectable Nothing-at-Stake allows the attacker to (undetectably) produce a greater fraction of blocks. The main takeaway from this section is that Recent Longest-Chain Variant Protocols are vulnerable to Undetectable Nothing-at-Stake.

\section{DISCUSSION}

In summary, we've shown that even in an ideal model with perfect connectivity and no latency, every Longest-Chain-Variant-Proof-of-Stake protocol has some undesirable property. Below, we discuss possible fixes for the attacks enabled by these properties, draw conclusions and pose directions for future work.

Preventing predictable selfish mining. Preventing predictable selfish mining is challenging, but some clever ideas exist in the literature. At a high level, Fruitchains [21], Ouroboros [17], and Tezos [14] design protocols where blocks need to be "supported" once mined (eligibility to support is also proportional to stake), so one would not only need a majority of blocks mined but also a majority of "support tokens(/fruit)" in a given window to successfully selfish mine. Both Snow White [9] and Ouroboros [17] provide proofs that any deviation from their prescribed protocol can only provide a small $\varepsilon$ in additional mining rewards. However, [9] notes that it would be preferable 
for known attacks to be strictly disincentivized (more on this in the full version of this paper), and it remains open whether these reward schemes accomplish this.

In personal communication, Vitalik Buterin has proposed another possible solution: whenever there are two blocks of the same height, punish both miners (e.g. by claiming a deposit). This certainly provides some disincentive for selfish mining, since the only form of possible profit comes from orphaning another block of the same height, and this event is directly punished. Of course, there is also potential downside to this approach, as forks happen naturally, so honest miners will occasionally lose their deposits as well. Still, this seems like a very promising direction.

Preventing predictable double-spend. A simple defense specifically against predictable doublespend attacks is to accept long confirmation times (e.g. a transaction is not considered "finalized" by vendors until several blocks have been announced descending from the block containing the transaction). Our analysis (see the full version of this paper) indicates that several hundred blocks might be necessary, making it virtually impossible to have quick confirmation times in a predictable Proof-of-Stake protocol without further defenses. For example, the authors of the Ouroboros [17] Protocol (which is predictable) suggest using confirmation times of 148 minutes to defend against double spend attacks by an attacker controlling $40 \%$ of the stake when blocks are created at a rate of one per minute (and this is consistent with our analysis).

Defending against Undetectable Nothing-at-Stake. Existing literature proposes roughly three paradigms that attempt to defend against Undetectable Nothing-at-Stake. The most common defense in commercial protocols is to set $D$ very large (these protocols are therefore $D$-locally predictable for large $D$ ), and to use some form of "checkpointing" every $\leq D$ blocks. This "checkpointing" might be run externally by a trusted party, hard-coded into the protocol, or just a form of trust among network participants that they would never seriously consider a fork more than $D$ blocks back. In practice, there don't seem to have been any serious issues with this approach, but to our knowledge its security hasn't previously been rigorously analyzed.

Algorand proposes a different approach: instead of using a longest-chain variant, it uses a Byzantine consensus protocol. Under some network connectivity assumptions, they show that the probability of a fork is negligible. As such, any deviant behavior that results in a fork (such as Undetectable Nothing-at-Stake) can be readily recognized as malicious, and safely ignored.

Ethereum's Casper [1] proposes a third solution that they call "dunkles": punish every miner whose block winds up being orphaned (not a predecessor of the block maximizing $S(A)$ ). The high-level goal of this is to essentially copy the incentives from Proof-of-Work: if your block is orphaned, you still lose the electricity that went into mining it. By punishing the miner of every orphaned block, some honest miners will get punished just by bad luck, but it will also discourage attackers from mining off the longest chain. This seems like a promising direction, but there is currently no formal specification or rigorous evaluation of the proposal.

There's no reason for global predictability. There's a real tradeoff to explore between $D$-local predictability and $D$-recency (since one cannot avoid both, by definition). But there doesn't seem to be any benefit to global predictability, only the risk of stronger predictable-selfish-mining attacks. There also doesn't seem to be a black-box reduction stating that mixing any existing protocol properly with digital signatures removes global predictability (although this would be a great future result), but it seems likely that a clever use of digital signatures as in Algorand could modify most existing protocols to be no longer globally predictable with low cost.

Trusted External Randomness, Trusted Checkpointing, or Not? Our work shows that there is a fundamental difference between trusted external randomness and "internal pseudorandomness" derived pseudorandomly using the cryptocurrency itself. Essentially, the difference is that all 
internal pseudorandomness is in the end locally predictable or recent and therefore susceptible to some form of attack. External randomness (such as the NIST beacon) is not, and recall that protocols that use external randomness fail to satisfy Chain Dependence (so none of our results apply). Can such a protocol be the basis for a secure, incentive compatible Proof-of-Stake protocol? Even if the answer is yes, how does one resolve the (possibly just-as-challenging) issue of obtaining trusted randomness? ${ }^{11}$ Trusted checkpointing provides a cheap solution to Undetectable Nothing-at-Stake, and does seem simpler than trusted randomness. Can trusted checkpointing provide guarantees that are otherwise hard (or impossible) to come by?

Byzantine Consensus versus Longest-Chain Variants. With the exception of Algorand and Casper: the Friendly Finality Gadget, every proposal that the authors are aware of uses a longest-chain variant. The upside of longest-chain variants is that they are inherently robust to Eclipse attacks. Byzantine consensus protocols require some network connectivity assumptions in order to safely ignore messages sent too far in the past, and are less robust to Eclipse attacks. Are there provable limits to what can be achieved by longest-chain variants, necessitating the use of Byzantine Consensus? Or is it possible to achieve the same guarantees with a true Longest-Chain variant?

Rigorously and Transparently Evaluate Protocols in the Ideal Model. Our work shows that already it is quite challenging to design incentive compatible Longest-Chain Variants in the ideal model. Numerous reasonable ideas have been proposed to address the vulnerabilities arising from predictability and recency, but none have transparent yet rigorous analysis. We believe that the vulnerabilities we've uncovered are serious enough that future Proof-of-Stake proposals should include transparent proofs of how they defend against predictable double-spend, predictable selfish-mine, and undetectable nothing-at-stake in the ideal model.

It is obviously necessary to also continue evaluating network security aspects of proposed protocols, but it is important that any incentive-related security claims in these rich models easily map to transparent claims in the ideal model.

\section{REFERENCES}

[1] 2017. Ethereum Wiki: Proof-of-Stake FAQ. https://github.com/ethereum/wiki/wiki/Proof-of-Stake-FAQ.

[2] Moshe Babaioff, Shahar Dobzinski, Sigal Oren, and Aviv Zohar. 2012. On bitcoin and red balloons. In Proceedings of the 13th ACM conference on electronic commerce. ACM, 56-73.

[3] Jonah Brown-Cohen, Arvind Narayanan, Christos-Alexandros Psomas, and S. Matthew Weinberg. 2018. Formal Barriers to Longest-Chain Proof-of-Stake Protocols. CoRR abs/1809.06528 (2018). arXiv:1809.06528 http://arxiv.org/ abs/1809.06528

[4] Vitalik Buterin and Virgil Griffith. 2017. Casper: The Friendly Finality Gadget.

[5] Miles Carlsten, Harry Kalodner, S Matthew Weinberg, and Arvind Narayanan. 2016. On the instability of bitcoin without the block reward. In Proceedings of the 2016 ACM SIGSAC Conference on Computer and Communications Security. ACM, 154-167.

[6] Jing Chen and Silvio Micali. 2016. ALGORAND: the efficient and democratic ledger. arXiv preprint arXiv:1607.01341 (2016).

[7] Nxt Community. 2014. Nxt Whitepaper. https://bravenewcoin.com/assets/Whitepapers/NxtWhitepaper-v122-rev4.pdf.

[8] NEM Community. 2015. NEM Technical Reference. https://nem.io/wp-content/themes/nem/files/NEM_techRef.pdf.

[9] Phil Daian, Rafael Pass, and Elaine Shi. 2016. Snow White: Provably Secure Proofs of Stake. IACR Cryptology ePrint Archive 2016 (2016), 919.

[10] Ittay Eyal. 2015. The Miner's Dilemma. In Proceedings of the 36th IEEE Symposium on Security and Privacy (Oakland).

[11] Ittay Eyal and Emin Gün Sirer. 2014. Majority is not enough: Bitcoin mining is vulnerable. In International conference on financial cryptography and data security. Springer, 436-454.

[12] Michael J. Fischer, Nancy Lynch, and Michael S. Paterson. 1985. Impossibility of Distributed Consensus with One Faulty Process. F. ACM 32, 2 (1985), 374-382.

\footnotetext{
${ }^{11}$ Chapter 9.4 of [20] describes how functional cryptocurrencies can serve as sources of trusted randomness. So it is indeed unclear whether trusted randomness is really an easier problem.
} 


\section{EC'19 Session 4b: Cryptocurrency and Financial Markets}

[13] Yossi Gilad, Rotem Hemo, Silvio Micali, Georgios Vlachos, and Nickolai Zeldovich. 2017. Algorand: Scaling Byzantine Agreements for Cryptocurrencies. In Proceedings of the 26th Symposium on Operating Systems Principles (SOSP '17) ACM, New York, NY, USA, 51-68. https://doi.org/10.1145/3132747.3132757

[14] L.M Goodman. 2014. Tezos A Self-Amending Crypto-Ledger White Paper. https://www.tezos.com/static/papers/ white_paper.pdf.

[15] Hanna Halaburda and Guillaume Haeringer. 2018. Bitcoin and Blockchain: What We Know and What Questions are Still Open. NYU Stern School of Business, Forthcoming (2018).

[16] Aggelos Kiayias, Elias Koutsoupias, Maria Kyropoulou, and Yiannis Tselekounis. 2016. Blockchain mining games. In Proceedings of the 2016 ACM Conference on Economics and Computation. ACM, 365-382.

[17] Aggelos Kiayias, Alexander Russell, Bernardo David, and Roman Oliynykov. 2017. Ouroboros: A provably secure proof-of-stake blockchain protocol. In Annual International Cryptology Conference. Springer, 357-388.

[18] Sunny King and Scott Nadal. 2012. PPCoin: Peer-to-Peer Crypto-Currency with Proof-of-Stake. https://peercoin.net/ assets/paper/peercoin-paper.pdf.

[19] Satoshi Nakamoto. 2008. Bitcoin: A peer-to-peer electronic cash system.

[20] Arvind Narayanan, Joseph Bonneau, Edward Felten, Andrew Miller, and Steven Goldfeder. 2016. Bitcoin and Cryptocurrency Technologies: A Comprehensive Introduction. Princeton University Press.

[21] Rafael Pass and Elaine Shi. 2017. Fruitchains: A fair blockchain. In Proceedings of the ACM Symposium on Principles of Distributed Computing. ACM, 315-324.

[22] Fahad Saleh. 2018. Blockchain without waste: Proof-of-stake. (2018).

[23] Ayelet Sapirshtein, Yonatan Sompolinsky, and Aviv Zohar. 2016. Optimal selfish mining strategies in bitcoin. In International Conference on Financial Cryptography and Data Security. Springer, 515-532.

[24] Yonatan Sompolinsky and Aviv Zohar. 2015. Secure high-rate transaction processing in bitcoin. In International Conference on Financial Cryptography and Data Security. Springer, 507-527.

[25] Pavel Vasin. 2014. BlackcoinâĂŹs proof-of-stake protocol v2. 